\title{
Patrón de sangrado uterino en adolescentes usuarias de implante anticonceptivo subdérmico de etonogestrel
}

\author{
Ingrid Leal F.1a ${ }^{1 a}$ Temístocles Molina ${ }^{1 b}$, Adela Montero ${ }^{1 c}$, Carolina González ${ }^{1}$, \\ Anna Macintyre. ${ }^{d}$ \\ ${ }^{1}$ Centro de Medicina y Desarrollo Integral de la Adolescencia (CEMERA), Facultad de Medicina, Universidad de Chile, \\ Santiago, Chile.
} aMatrona, Magíster en Salud Pública. ${ }^{b}$ Bioestadístico, Magíster en Bioestadística. ${ }^{c}$ Magíster en Bioética. ${ }^{d}$ Enfermera,
Master in International Community Health.

\section{RESUMEN}

Objetivo: Describir las características del patrón sangrado uterino de las adolescentes que usan el implante anticonceptivo sudérmico de etonogestrel (IASE) que concurren a un centro especializado en salud sexual y reproductiva de adolescentes en Santiago, Chile, y su asociación con variables biopsicosocial. Método: Estudio de cohorte retrospectiva de las usuarias de IASE. Los datos fueron analizados utilizando la ecuación de estimación generalizada, análisis de sobrevida de Kaplan Meier y test de log-rank. Resultados: La cohorte incluyó a 62 adolescentes. La media de edad fue de 16,2 años. Las participantes recibieron asesoramiento anticonceptivo antes de la inserción del IASE, y fueron seguidas durante tres años. Los patrones de sangrado más frecuentes durante el primer año fue el de amenorrea $(40,5 \%)$ y el sangrado aceptable $(27,1 \%)$, mientras que el menos frecuente fue prolongado y/o sangrado frecuente $(15,8 \%)$. De acuerdo con el análisis de sobrevida, la única variable biopsicosocial asociado con patrón prolongado y/o sangrado frecuente fue condición médica previa. Cinco adolescentes (8\%) se retiraron el IASE. Conclusiones: EI IASE es una opción anticonceptiva segura y altamente eficaz para las adolescentes, independientemente de la paridad. La consejería es de gran importancia para fomentar la tolerancia y la adhesión al implante.

\section{PALABRAS CLAVE: Implantes anticonceptivos, servicios de salud para adolescentes, salud reproductiva}

\section{SUMMARY}

Objectives: To describe uterine bleeding patterns of adolescents using the long term etonogestrel contraceptive implant (ENG implant) attending a specialized adolescent sexual and reproductive health centre in Santiago, Chile, and test their association with bio-psychosocial variables. Methods: A retrospective cohort study of ENG implant users was conducted and data were analysed using the Generalized Estimating Equation, Kaplan Meier Survival Analysis and Log-Rank Test. Results: The cohort included 62 adolescents with and average age at inclusion of 16.2 years. Participants received contraceptive counselling prior to insertion of an ENG implant, and were followed up for three years. The most frequent bleeding patterns during the first year were amenorrhea (40.5\%) and acceptable bleeding (27.1\%), whilst the least frequent was prolonged and/or frequent bleeding (15.8\%). According to the survival analysis, the only bio-psychosocial variable associated with prolonged and/or frequent bleeding was prior medical condition. Five adolescents 
$(8 \%)$ withdrew from the treatment. Conclusions: The ENG implant is a safe and highly effective contraceptive option for adolescents, regardless of parity. Counselling is of great importance to foster tolerance and adherence to the ENG implant.

\section{KEY WORDS: Contraception, contraceptive implants, adolescent health services, reproductive health}

\section{INTRODUCCIÓN}

En Chile durante el año 2012 hubo 35.184 nacimientos de hijos de madres menores de 19 años de edad, incluyendo 874 nacimientos de adolescentes menores de 15 años de edad. Las estadísticas muestran que la edad de iniciación sexual en Chile ha ido disminuyendo siendo menor en mujeres de bajo nivel socioeconómico (1). Existe evidencia que las adolescentes, a pesar de conocer la existencia de métodos anticonceptivos y lugares a los que pueden acceder a estos, no consultan a los centros de salud debido a múltiples barreras de los sistemas de salud y la falta o insuficiencia de educación sexual (2).

Según el Ministerio de Salud durante el año $2011,168.773$ adolescentes asistieron a control para regulación de la fertilidad en el sistema de salud público, incluyendo 6.917 adolescentes menores de 15 años de edad. Los anticonceptivos combinados orales fueron el método más utilizados $(47 \%)$ seguidos de los anticonceptivos inyectables combinados (16\%) (3).

En cuanto al tipo y la continuidad del uso de anticonceptivos, es importante tener en cuenta que las mayores tasas de fracaso anticonceptivo y las pérdidas de seguimiento se han observado en las adolescentes en comparación con las mujeres adultas, especialmente con los anticonceptivos que requieren ingesta diaria o alta motivación de las usuarias, tales como píldoras anticonceptivos orales e inyectables mensuales (4).

El Colegio Americano de Obstetras y Ginecólogos recomienda para las adolescentes utilizar anticonceptivos reversibles de acción prolongada (LARCs=Long-Acting reversible contraception), tales como los dispositivos intrauterinos y los implantes anticonceptivos, ya que estos anticonceptivos tienen mayores tasas de continuidad, eficacia y satisfacción de las usuarias, en comparación con los anticonceptivos orales e inyectables combinados (5). El implante anticonceptivo sudérmico de etonogestrel (IASE) es un LARC que funciona mediante la inhibición de la ovulación, mientras que también aumenta la viscosidad de la mucosa cervical, di- ficultando el paso de los espermatozoides por el canal endocervical $(6,7)$.

Los estudios han demostrado que el IASE es eficaz en la prevención tanto del primer embarazo como del segundo embarazo durante la adolescencia, debido a las mayores tasas de continuidad en comparación con los anticonceptivos de acción corta, por su prolongada duración (tres años) y uso independiente de la usuaria $(8,9)$. Sin embargo, el IASE tiene efectos secundarios, como sangrado prolongados, siendo la causa principal causa de abandono del tratamiento $(10,11,12)$. El patrón de sangrado durante los primeros tres meses es altamente predictivo de futuros patrones de sangrado. Aproximadamente un tercio de las usuarias experimentaran sangrado normal o menos frecuente y un $20 \%$ experimentara sangrados prolongados $\mathrm{y} / \mathrm{o}$ más frecuentes $(12,13)$.

El asesoramiento antes de la inserción del IASE es la principal estrategia para promover la continuidad de uso del implante, así como el manejo médico de los sangrados prolongados a través de la prescripción de anticonceptivos orales combinados y/o antiinflamatorios no esteroidales (5). Otros regímenes descritos para tratar el sangrado prolongado es el uso de mifepristona y tetraciclina, sin embargo estos no se utilizan en nuestro medio (14). Las reacciones adversas más comunes al IASE son cefaleas, acné, mastalgia, dismenorrea y aumento de peso $(8,15)$.

Del número total de adolescentes que asistieron a control para regulación de la fertilidad en el sistema público de salud durante el año en 2011, $6,9 \%$ eran usuarias de implante (3). La experiencia con adolescentes sugiere que este método anticonceptivo es más frecuentemente indicado para las madres adolescentes para prevenir un segundo embarazo $(9,16)$ a pesar de que el Colegio Americano de Obstetras y Ginecólogos recomiendan el uso del implante, independientemente de la paridad (5).

El objetivo de este estudio fue describir y comparar los patrones de sangrado en las usuarias adolescentes de IASE en relación con las características biopsicosocial. 


\section{PACIENTES Y MÉTODO}

La población del estudio consistió en una cohorte histórica de las usuarias adolescentes de IASE que asistieron a un centro especializado en salud sexual y reproductiva en Santiago de Chile, entre septiembre de 2007 y julio de 2012. De acuerdo con este diseño, los datos basales y de seguimiento fueron recolectados de forma retrospectiva. Las adolescentes pertenecen a la región metropolitana de Chile y corresponden al nivel socioeconómico medio y medio bajo. Para ser incluidas en el estudio, la inserción del IASE debió realizarse en el mismo centro, quedando excluidas aquellas adolescentes cuyo IASE fue insertado en otro centro de salud. La consejería para la elección del anticonceptivo se realizó antes de la inserción del IASE. Los patrones de sangrado fueron monitoreados cada tres meses usando un calendario menstrual que se clasifica en los siguientes patrones: amenorrea, sangrado infrecuente, sangrado frecuente, sangrado prolongado, sangrado irregular y sangrado aceptable (definiciones en la Tabla I) (17). Las variables independientes (variables biopsicosociales) fueron: edad, edad de la menarquia, inicio de actividad sexual, edad de la primera relación sexual, uso de anticonceptivos anterior, paridad, patología médica, patología de salud mental, peso inicial y estado nutricional. Para analizar la relación entre patrón de sangrado y las variables biopsicosociales controlado por el tiempo de uso del método, se utilizaron modelos GEE (Ecuación de Estimación Generalizada) (18).

Como se ha descrito anteriormente, una de las causas de abandono del uso del IASE es la presencia de patrones de sangrado prolongados y/o frecuentes, por lo tanto, la función de sobrevida de

\section{Tabla I}

\section{DEFINICIÓN DE PATRÓN DE SANGRADOS EN UN PERIODO DE 90 DÍAS DE REFERENCIA}

\begin{tabular}{ll}
\hline Condición & Definición \\
\hline $\begin{array}{l}\text { Amenorrea } \\
\begin{array}{l}\text { Sangrado } \\
\text { frecuente }\end{array}\end{array}$ & Ausencia de sangrado o goteo \\
$\begin{array}{l}\text { Sangrado } \\
\text { infrecuente }\end{array}$ & $1-2$ episodios de sangrado o goteo \\
$\begin{array}{l}\text { Sangrado } \\
\text { prolongado }\end{array}$ & $\begin{array}{l}\geq 1 \text { sangrados-goteo que dura más } \\
\text { de } 14 \text { días }\end{array}$ \\
$\begin{array}{l}\text { Sangrado } \\
\text { aceptable }\end{array}$ & Ninguno de los patrones anteriores \\
\hline
\end{tabular}

estos patrones de sangrado se evaluó mediante el análisis de sobrevida de Kaplan-Meier (19). Para este análisis estadístico, los patrones de sangrado se dicotomizaron en los patrones de sangrado prolongado $\mathrm{y} / \mathrm{o}$ frecuentes $=1 \mathrm{y}$ otros patrones de sangrado $=0$. Este análisis calcula la probabilidad de sobrevida para este patrón de sangrado en un período de tiempo definido, en otras palabras, la probabilidad de que una usuaria no presente sangrados prolongados y/o frecuentes dentro de un número definido de meses. Se analizaron las variables biopsicosocial medidas al inicio del estudio para evaluar la asociación con estos tiempos de sobrevida mediante el test log-rank con un nivel de significación de $p<0,05$ (19).

El estudio fue aprobado por el Comité de Ética de Investigación de la Facultad de Medicina, Universidad de Chile. El consentimiento informado se obtuvo de todas las participantes antes de la consejería anticonceptiva en el centro de salud y todos los datos de las pacientes se recogieron de forma anónima en el programa Excel.

\section{RESULTADOS}

Durante el período de estudio, el IASE se indicó a 62 adolescentes. La edad mediana de edad al momento de la inserción fue de 16,2 años (rango: $13-20$ años). El 88,7\% de las adolescentes había iniciado actividad sexual, con una edad media de 14,3 años. El $77,4 \%$ había utilizado anticonceptivos previamente, siendo los más comunes los anticonceptivos inyectables combinados mensuales (37,1\%). El $74,2 \%$ eran nuligestas; $25,8 \%$ informó alguna patología médica (migrañas, síndrome metabólico, cardiopatías, infección por VIH), mientras que el 58,1\% informó de alguna patología de salud mental (trastorno del estado de ánimo, disminución de la capacidad cognitiva, consumo problemático de alcohol y/o drogas). El estado nutricional al inicio del estudio mostró que el $44,8 \%$ eran obesas o con sobrepeso, con una adolescente que pesaba más de 90 kilos (Tablas II y III).

En relación al seguimiento, el 37,3\% completó los tres años, el $17 \%$ completó entre dos y tres años, el $22 \%$ completó entre uno y dos años, y el $23,7 \%$ completo menos de un año de seguimiento.

No se reportaron fallas anticonceptivos con el IASE. Cinco implantes se extrajeron de forma prematura ( $8 \%)$, dos debido a deseo de embarazo, dos debido a reacciones adversas (aumento de peso y acné) y uno por sangrado prolongado que no respondió al tratamiento. Sólo un implante fue retirado antes de completar un año de uso. Después de los tres años de seguimiento, 15 adolescentes (24\%) recibieron un nuevo IASE. 
Tabla II

CARACTERÍSTICAS DE ADOLESCENTES USUARIAS DE IASE

\begin{tabular}{|c|c|c|}
\hline Características & $\mathrm{n}$ & $\%$ \\
\hline \multicolumn{3}{|l|}{ Inicio de actividad sexual } \\
\hline $\mathrm{Si}$ & 55 & 88,7 \\
\hline No & 7 & 11,3 \\
\hline \multicolumn{3}{|l|}{ Anticonceptivo previo } \\
\hline Píldoras anticonceptivas combinadas de progestágenos solos & 12 & 19,3 \\
\hline Inyectables combinados & 23 & 37,1 \\
\hline Acetato de medroxiprogesterona de depósito & 2 & 3,2 \\
\hline Dispositivo intrauterino & 1 & 1,6 \\
\hline Condón & 10 & 16,1 \\
\hline Ninguno & 14 & 22,7 \\
\hline \multicolumn{3}{|l|}{ Embarazos Previos } \\
\hline Nuligesta & 45 & 72,6 \\
\hline Nulípara & 1 & 1,6 \\
\hline Multípara & 16 & 25,8 \\
\hline \multicolumn{3}{|l|}{ Patología médica } \\
\hline No & 46 & 74,2 \\
\hline $\mathrm{Si}$ & 16 & 25,8 \\
\hline \multicolumn{3}{|l|}{ Patología salud mental } \\
\hline No & 26 & 41,9 \\
\hline $\mathrm{Si}$ & 36 & 58,1 \\
\hline \multicolumn{3}{|l|}{ Estado nutricional inicial } \\
\hline Bajo peso & 2 & 3,5 \\
\hline Normal & 30 & 51,7 \\
\hline Sobrepeso & 17 & 29,3 \\
\hline Obesa & 9 & 15,5 \\
\hline
\end{tabular}

Tabla III

CARACTERÍSTICAS DE ADOLESCENTES USUARIAS DE IASE

\begin{tabular}{lccccc}
\hline Características & $\mathrm{n}$ & Promedio & Mediana & \pm DE & Rango \\
\hline Edad de inserción (años) & 62 & 16,2 & 16 & 1,5 & $13-20$ \\
Edad de menarquia (años) & 62 & 11,7 & 12 & 1,6 & $8-16$ \\
Edad inicio sexual (años) & 55 & 14,3 & 14 & 1,7 & $11-19$ \\
Peso inicial (kg) & 59 & 59,1 & 56,4 & 11,4 & $42,7-93,0$ \\
\hline
\end{tabular}

Durante los tres primeros meses, los patrones de sangrado aceptable y amenorrea se presentaron en el $28,9 \%$ de las usuarias, respectivamente (Tabla IV). Entre tres y seis meses, el 41,7\% experimentó amenorrea y patrones de sangrado aceptable $30,5 \%$. Entre los seis y nueve meses, el $48,3 \%$ presentó amenorrea y el 17,2\% sangrado aceptable. Por último, entre los nueve y doce meses, el
42,9\% experimentó amenorrea y el 32,1\% sangrado aceptable. El patrón de sangrado prolongado y/o sangrado frecuente se presentó en el 17,8\% de las usuarios en los tres primeros meses, el 13,9\% entre los tres a seis meses, el $17,2 \%$ entre los seis y nueve meses, y el 14,3\% entre los nueve y doce meses. 


\section{Tabla IV \\ TIPO DE PATRÓN DE SANGRADO REGISTRADO EN INTERVALOS DE TRES MESES DURANTE 36 MESES}

\begin{tabular}{|c|c|c|c|c|c|c|c|c|c|c|c|c|}
\hline \multirow{3}{*}{$\begin{array}{l}\text { Patrón de } \\
\text { sangrado }\end{array}$} & \multicolumn{12}{|c|}{ MESES } \\
\hline & 3 & 6 & 9 & 12 & 15 & 18 & 21 & 24 & 27 & 30 & 33 & 36 \\
\hline & $\begin{array}{c}n \\
(\%)\end{array}$ & $\begin{array}{c}\mathrm{n} \\
(\%)\end{array}$ & $\begin{array}{c}n \\
(\%)\end{array}$ & $\begin{array}{c}n \\
(\%)\end{array}$ & $\begin{array}{c}n \\
(\%)\end{array}$ & $\begin{array}{l}\mathrm{n} \\
(\%)\end{array}$ & $\begin{array}{c}\mathrm{n} \\
(\%)\end{array}$ & $\begin{array}{c}n \\
(\%)\end{array}$ & $\begin{array}{c}n \\
(\%)\end{array}$ & $\begin{array}{c}n \\
(\%)\end{array}$ & $\begin{array}{c}\mathrm{n} \\
(\%)\end{array}$ & $\begin{array}{c}n \\
(\%)\end{array}$ \\
\hline Amenorrea & $\begin{array}{c}13 \\
(28,9)\end{array}$ & $\begin{array}{c}15 \\
(41,7)\end{array}$ & $\begin{array}{c}14 \\
(48,3)\end{array}$ & $\begin{array}{c}12 \\
(42,9)\end{array}$ & $\begin{array}{c}7 \\
(41,2)\end{array}$ & $\begin{array}{c}8 \\
(34,8)\end{array}$ & $\begin{array}{c}6 \\
(37,5)\end{array}$ & $\begin{array}{c}6 \\
(35,3)\end{array}$ & $\begin{array}{c}2 \\
(18,2)\end{array}$ & $\begin{array}{c}3 \\
(37,5)\end{array}$ & $\begin{array}{c}2 \\
(22,2)\end{array}$ & $\begin{array}{c}6 \\
(27,3)\end{array}$ \\
\hline $\begin{array}{l}\text { Prolongado o } \\
\text { frecuente }\end{array}$ & $\begin{array}{c}8 \\
(17,8)\end{array}$ & $\begin{array}{c}5 \\
(13,9)\end{array}$ & $\begin{array}{c}5 \\
(17,2)\end{array}$ & $\begin{array}{c}4 \\
(14,3)\end{array}$ & $\begin{array}{c}3 \\
(17,7)\end{array}$ & $\begin{array}{c}3 \\
(13,0)\end{array}$ & $\begin{array}{c}2 \\
(12,5)\end{array}$ & $\begin{array}{c}3 \\
(17,7)\end{array}$ & $\begin{array}{c}5 \\
(45,4)\end{array}$ & $\begin{array}{c}1 \\
(12,5)\end{array}$ & - & $\begin{array}{c}3 \\
(13,6)\end{array}$ \\
\hline Infrecuente & $\begin{array}{c}11 \\
(24,4)\end{array}$ & $\begin{array}{c}5 \\
(13,9)\end{array}$ & $\begin{array}{c}5 \\
(17,2)\end{array}$ & $\begin{array}{c}3 \\
(10,7)\end{array}$ & $\begin{array}{c}4 \\
(23,5)\end{array}$ & $\begin{array}{c}5 \\
(21,8)\end{array}$ & $\begin{array}{c}2 \\
(12,5)\end{array}$ & $\begin{array}{c}4 \\
(23,5)\end{array}$ & $\begin{array}{c}1 \\
(9,1)\end{array}$ & $\begin{array}{c}2 \\
(25,0)\end{array}$ & $\begin{array}{c}3 \\
(33,3)\end{array}$ & $\begin{array}{c}5 \\
(22,7)\end{array}$ \\
\hline Aceptable & $\begin{array}{c}13 \\
(28,9)\end{array}$ & $\begin{array}{c}11 \\
(30,5)\end{array}$ & $\begin{array}{c}5 \\
(17,2)\end{array}$ & $\begin{array}{c}9 \\
(32,1)\end{array}$ & $\begin{array}{c}3 \\
(17,7)\end{array}$ & $\begin{array}{c}7 \\
(30,4)\end{array}$ & $\begin{array}{c}6 \\
(37,5)\end{array}$ & $\begin{array}{c}4 \\
(23,5)\end{array}$ & $\begin{array}{c}3 \\
(27,3)\end{array}$ & $\begin{array}{c}2 \\
(25,0)\end{array}$ & $\begin{array}{c}4 \\
(44,5)\end{array}$ & $\begin{array}{c}8 \\
(36,4)\end{array}$ \\
\hline Total & $\begin{array}{c}45 \\
(100)\end{array}$ & $\begin{array}{c}36 \\
(100)\end{array}$ & $\begin{array}{c}29 \\
(100)\end{array}$ & $\begin{array}{c}28 \\
(100)\end{array}$ & $\begin{array}{c}17 \\
(100)\end{array}$ & $\begin{array}{c}23 \\
(100)\end{array}$ & $\begin{array}{c}16 \\
(100)\end{array}$ & $\begin{array}{c}17 \\
(100)\end{array}$ & $\begin{array}{c}11 \\
(100)\end{array}$ & $\begin{array}{c}8 \\
(100)\end{array}$ & $\begin{array}{c}9 \\
(100)\end{array}$ & $\begin{array}{c}22 \\
(100)\end{array}$ \\
\hline Inasistentes ${ }^{*}$ & 17 & 26 & 33 & 34 & 45 & 39 & 46 & 45 & 51 & 54 & 53 & 40 \\
\hline
\end{tabular}

*Corresponde al número de usuarias que no se presentaron al control programado

Durante el período de seguimiento en promedio se presentaron los siguiente patrones de sangrado: $34,7 \%$ amenorrea, $29,3 \%$ sangrado aceptable, $19,8 \%$ sangrado infrecuente y el $16,3 \%$ el sangrado prolongado y/o frecuente.

En relación con el tratamiento médico para los sangrados prolongados, de las 19 adolescentes que experimentaron este patrón, 15 requirieron de tratamiento con píldoras combinadas de levonorgestrel o desogestrel y/o anti-inflamatorios no esteroidales. No hubo nuevos casos de sangrado prolongado y/o frecuente después de los 27 meses de seguimiento.

De acuerdo con el análisis de sobrevida, el $61 \%$ de las adolescentes no experimentó sangrado prolongado y/o frecuente después del mes 27 de uso del IASE (Tabla V). En adolescentes sin patología médica, el $49,1 \%$ no presentó sangrado prolongado y/o frecuente después de 27 meses de uso, mientras que para las adolescentes con antecedentes de patología médica, ninguna presento sangrado prolongado y/o sangrado frecuente $(p=0,005)$. Ninguna de las variables biopsicosocial restantes mostró asociación estadísticamente significativa con los patrones de sangrado.

\section{DISCUSIÓN}

En nuestro estudio el $72,6 \%$ de las adolescentes inició el uso del IASE para la prevención del primer embarazo, lo cual se diferencia de otros estudios
Tabla V

DESCRIPCIÓN DEL TIEMPO DE SOBREVIDA PARA EL PATRÓN DE SANGRADO PROLONGADO Y/O FRECUENTE

\begin{tabular}{ccc}
\hline Tiempo (meses) & $\begin{array}{c}\text { Función de } \\
\text { sobrevida }\end{array}$ & $\begin{array}{c}\text { Intervalo de } \\
\text { confianza } 95 \%\end{array}$ \\
\hline 3 & 0,86 & $0,75-0,93$ \\
6 & 0,75 & $0,71-0,90$ \\
9 & 0,72 & $0,61-0,84$ \\
12 & 0,72 & $0,59-0,83$ \\
15 & 0,72 & $0,59-0,83$ \\
18 & 0,67 & $0,53-0,79$ \\
21 & 0,67 & $0,53-0,79$ \\
24 & 0,64 & $0,50-0,77$ \\
27 & 0,61 & $0,45-0,74$ \\
30 & 0,61 & $0,45-0,74$ \\
33 & 0,61 & $0,45-0,74$ \\
36 & 0,61 & $0,45-0,74$ \\
\hline
\end{tabular}

en los que la mayoría de las adolescentes utilizan el implante para la prevención de un segundo embarazo $(9,10,16)$. Es importante destacar que las adolescentes nuligestas son elegibles para la anticoncepción de largo plazo, de acuerdo a las recomendaciones de las sociedades científicas, por su 
alta eficiencia, seguridad e independencia de uso de la usuaria (5). La edad promedio al momento de la inserción fue de 16,2 años, la cual es menor a la reportada en otros estudios, esto probablemente sugiere la necesidad de prevención del embarazo a edades más tempranas en Chile $(9,16)$.

Es destacable que el $11,3 \%$ de las usuarias inició el uso del IASE antes del inicio de la actividad sexual y que para el $38,8 \%$ de las adolescentes se trataba de su primer anticonceptivo hormonal. En adolescentes que habían usado anticonceptivos hormonales anteriormente, el anticonceptivo más frecuentemente usado fueron los anticonceptivos inyectables combinados. El frecuente uso de anticonceptivos inyectables en nuestra muestra difiere de lo descrito a nivel nacional e internacional, donde los anticonceptivos más utilizados en la adolescencia son las píldoras anticonceptivas combinadas $(3,8,11)$.

Una característica de esta población fue la alta prevalencia de patología médica y de salud mental, que muy probablemente influyeron en la selección de este anticonceptivo para estas usuarias. El principal obstáculo para acceder al IASE en Chile es la barrera de disponibilidad o económica, por lo tanto, estos implantes han sido priorizados para adolescentes de alto riesgo. Esta situación está cambiando debido a un mayor acceso a distintas opciones anticonceptivas, usuarias más informadas y asesoradas, lo que permite a niñas y mujeres jóvenes a elegir anticonceptivos que proporcionan una mayor independencia y mayor eficacia $(11,20,21,22)$. Esta tendencia coincide con las actuales recomendaciones sobre el uso de LARCs en adolescentes sanas y también para aquellas con necesidades especiales, como los adolescentes con antecedentes de enfermedades crónicas $(5,19)$.

$\mathrm{Al}$ igual que en otro estudios, los patrones de sangrado más frecuente en el primer año fueron amenorrea $(40,5 \%)$, sangrado aceptable $(27,1 \%)$ y sangrado infrecuente $(19,8 \%)(12,13,16)$. Este resultado es positivo, teniendo en cuenta que estos patrones de sangrado tienden a ser mejor tolerados que los sangrados prolongados y/o sangrados frecuentes, contribuyendo así a una mayor adhesión al método anticonceptivo.

Nuestro estudio encontró que en promedio el $15,8 \%$ de las usuarias experimentaron sangrado prolongado durante el primer año de uso, un resultado similar al que se describe en una revisión de 11 estudios, que mostró una frecuencia de 17\% para el sangrado prolongado (13). Sin embargo, otro estudio con madres adolescentes informó sólo 5\% de frecuencia de sangrado prolongado durante el primer año de uso (16). Estas diferencias no se asociaron con las características de las usuarias, tales como la paridad o la edad (16). Además, otro estudio concluyó que los patrones de sangrados no pueden predecirse por estas variables biopsicosocial (23).
En el presente estudio se encontró diferencias en la presencia de patrones de sangrado prolongados y/o frecuentes cuando se comparan las adolescentes con y sin patología médica, sin embargo, dada la heterogeneidad de estas condiciones (migrañas, síndrome metabólico, patología cardiaca, la infección por $\mathrm{VIH}$ ), no es posible determinar el mecanismo que esté detrás de esta asociación.

Desde una perspectiva clínica, el hecho de que las adolescentes con patología médica previa no presentaron hemorragia prolongada y/o frecuente fue favorable, ya que el manejo médico de sangrados prolongados es más complejo en las usuarias con condiciones médicas, en las que el uso de estrógenos, por ejemplo, están contraindicados, como es en el caso de adolescentes con cardiopatías (17).

Al igual que en otros estudios, se trataron los casos de sangrado prolongado a través consejería y la prescripción de píldoras anticonceptivas combinadas (desogestrel o levonorgestrel) y/o antiinflamatorios no esteroidales $(13,15,24)$. Destacamos la consejería realizada, para la promoción de la tolerancia a los diferentes patrones de sangrados. Estas intervenciones han contribuido de manera significativa a la reducción de los retiros prematuros del IASE. De las adolescentes que presentaron sangrado prolongado, sólo una solicitó el retiro anticipado del implante, a diferencia de otros estudios que reportan hasta $23 \%$ de retiros durante el primer año debido a los sangrados prolongados $(12,23)$.

Las limitaciones de este estudio están relacionadas con su diseño. Al tratarse de un estudio de cohorte retrospectiva, no fue posible que los investigadores controlaran la asistencia de las participantes para el seguimiento de los controles. Tres adolescentes no regresaron para el seguimiento después de la inserción del implante, lo que probablemente se asocia con la presencia de características de alto riesgo (alcohol, drogas, comorbilidades).

Nuestro centro es una institución especializada en la atención en salud sexual y reproductiva para adolescentes, en particular adolescentes de mayor vulnerabilidad social y económica. En 2007 iniciamos nuestro trabajo con el IASE con resultados altamente favorables, incluidas la baja frecuencia de retiros prematuros, muy probablemente debido a la consejería realizada previo a la inserción y durante el seguimiento. Sin embargo, para el año 2014 las usuarias de IASE representaban sólo el $10 \%$ de todas las usuarias de anticonceptivos en nuestro centro, esto atribuido principalmente a que disponemos de un número limitado de implantes para ofrecer a las adolescentes consultantes en nuestro centro y a las barreras económicas, que impiden que ellas puedan acceder al implante a través de la compra directa. Se han descrito en otros lugares, otras barreras para el uso del IASE dentro 
de las cuales se identifican, la falta de capacitación y las actitudes negativas de algunos profesionales de la salud con respecto a la sexualidad de los adolescentes y la anticoncepción, siendo las principales razones para el bajo uso de implantes (25).

\section{CONCLUSIÓN}

EI IASE es una opción anticonceptiva segura y eficaz para los adolescentes, especialmente las que tienen un mayor grado de vulnerabilidad, independientemente de la paridad. La consejería es muy importante para promover la tolerancia a los patrones de sangrado y el manejo clínico adecuado cuando se presentan sangrados prolongados, asegurando así la continuidad y adherencia al método anticonceptivo.

\section{REFERENCIAS}

1. Instituto Nacional de Estadísticas. Anuario de Estadísticas Vitales 2013. Santiago, 2015.

2. Sadler M, Obach A, Luengo X, et al: Estudio de barreras de acceso a los servicios de salud para la prevención del embarazo adolescente en Chile. Santiago, Chile, MINSAL, 2011. Disponible en: http://web. minsal.cl/portal/url/item/ace74d077631463de040010 11e011b94.pdf.

3. Departamento de Ciclo Vital: Situación actual del embarazo adolescente en Chile. Santiago, Chile, MINSAL, 2012. Disponible en: http://portales.mineduc.cl/usuarios/convivencia_escolar/doc/201210251259030.situacion_actual_embarazo_adolescente_en_chile.pdf.

4. González E, Molina T, Montero A, Martínez V, Molina $R$. Factores asociados a la continuidad del uso anticonceptivo en adolescentes solteras nulíparas. Rev Méd Chile 2009;137(9):1187-92.

5. Committee on Adolescent Health Care Long-Acting Reversible Contraception Working Group, The American College of Obstetricians and Gynecologists. Committee opinion no. 539: adolescents and longacting reversible contraception: implants and intrauterine devices. Obstet Gynecol. 2012;120(4):983-8.

6. Mäkäräinen L, Van Beek A, Tuomivaara L, Aspelund $B$, Coelingh Bennink $H$. Ovarian function during the use of a single contraceptive implant: Implanon compared with Norplant. Fertil Steril 1998;69(4):714-21.

7. Peralta O, Diaz S, Croxatto H. Subdermal contraceptive implants. J. Steroid Biochem Molec Biol 1995;53(16):223-6.

8. Guazzelli CA, de Queiroz FT, Barbieri M, Torloni MR, de Araujo FF. Etonogestrel implant in adolescents: evaluation of clinical aspects. Contraception 2011;83(4):336-9.

9. Lewis LN, Doherty DA, Hickey M, Skinner SR. Implanon as a contraceptive choice for teenage mothers: a comparison of contraceptive choices, acceptability and repeat pregnancy. Contraception 2010;81(5):4216.

10. Tocce KM, Sheeder JL, Teal SB. Rapid repeat pregnancy in adolescents: do immediate postpartum contraceptive implants make a difference? Am J Obstet Gynecol 2012;206(6):481.e1-7.

11. Prescott GM, Matthews CM. Long-acting reversible contraception: a review in special populations. Pharmacotherapy 2014;34(1):46-59.

12. Deokar AM, Jackson W, Omar HA. Menstrual bleeding patterns in adolescents using etonogestrel (ENG) implant. Int J Adolesc Med Health 2011;23(1):75-7.

13. Mansour D, Bahamondes L, Critchley H, Darney P, Fraser IS. The management of unacceptable bleeding patterns in etonogestrel-releasing contraceptive implant users. Contraception 2011;83(3):202-10.

14. Abdel-Aleem $\mathrm{H}$, d'Arcangues $\mathrm{C}$, Vogelsong KM, Gaffield ML, Gülmezoglu AM. Treatment of vaginal bleeding irregularities induced by progestin only contraceptives. Cochrane Database Syst Rev. 2013 Oct 21;(10):CD003449

15. Adams K, Beal MW. Implanon: a review of the literature with recommendations for clinical management. J Midwifery Womens Health 2009;54(2):142-9.

16. Guazzelli CA, de Queiroz FT, Barbieri M, Torloni MR, de Araujo FF. Etonogestrel implant in postpartum adolescents: bleeding pattern, efficacy and discontinuation rate. Contraception 2010;82(3):256-9.

17. Organización Mundial de la Salud, Departamento de Salud Reproductiva e Investigaciones Conexas. Criterios médicos de elegibilidad para el uso de anticonceptivos, Cuarta edición, 2009. Disponible en: http://www.who.int/reproductivehealth/publications/ family_planning/9789241563888/es/.

18. Hardin JW, Hilbe JM. Generalized Estimating Equations. Boca Raton, FL, Chapman \& Hall/CRC, 2003.

19. Kaplan EL, Meier P. Nonparametric estimation from incomplete observations. J Am Stat Assoc 1958;53(282):457-81.

20. Amies Oelschlager AM, Micks EA, Debiec KE, Nizamic T, Mantrala MD, Prager SW. Long acting reversible contraception in adolescents with cardiovascular conditions. J Pediatr Adolesc Gynecol 2014; 27(6):353-5.

21. González RC, Montero A, Verges V, Leal I. Use of contraceptive implant (Implanon $®$ ) among chilean adolescents. J Pediatr Adolesc Gynecol 2011; 24(2):e70-1.

22. Spies EL, Askelson NM, Gelman E, Losch M. Young women's knowledge, attitudes, and behaviors related to long-acting reversible contraceptives. Womens Health Issues 2010;20(6):394-9.

23. Casey PM, Long ME, Marnach ML, Bury JE. Bleeding related to etonogestrel subdermal implant in a US population. Contraception 2011;83(5):426-30.

24. Weisberg E, Hickey M, Palmer D, O'Connor V, Salamonsen LA, Findlay JK, Fraser IS. A randomized controlled trial of treatment options for troublesome uterine bleeding in Implanon users. Hum Reprod 2009; 24(8):1852-61.

25. Leal I, Lüttges C, Troncoso P, Leyton C, Molina T, Eguiguren $\mathrm{P}$. Conocimientos y práctica clínica de los proveedores de salud para la prevención del embarazo adolescente según marco legal Chileno. Rev Med Chile 2016; 144(5): 577-84. 